\title{
La relación entre la discursividad y la no relacionalidad: un análisis basado en corpus
}

\author{
Aroa OrRequia Barea \\ Personal Técnico de Apoyo a la Investigación I+D+i \\ Instituto Universitario de Investigación en Lingüística Aplicada \\ Universidad de Cádiz \\ Avda. Duque de Nájera 16 \\ Edificio Simón Bolivar, $2^{\mathrm{a}}$ planta \\ 11002 Cádiz \\ E-mail: aroa.orrequia@uca.es
}

\section{LA RELACIÓN ENTRE LA DIS- CURSIVIDAD Y LA NO RELA- CIONALIDAD: UN ANÁLISIS BA- SADO EN CORPUS}

\begin{abstract}
RESUMEN: En inglés existe un conjunto de verbos completivos que se comporta de manera distinta al resto en lo que a su semántica y sintaxis se refiere. El objetivo de este trabajo es determinar cuál es la naturaleza de este comportamiento. Nuestra hipótesis es que las especificidades gramaticales de estos verbos derivan de su referencia al discurso, de ahi que los denominemos discursivos. Para demostrarlo empíricamente hemos realizado, basándonos en corpus, un análisis de los verbos completivos que pueden concurrir con construcciones en estilo directo. En primer lugar, hemos seleccionado las clasificaciones de verbos más relevantes de la literatura, para luego realizar una búsqueda de dichos verbos seguidos de una cita directa en el British National Corpus. Los resultados han venido a confirma nuestra hipótesis, ya que los verbos discursivos son los únicos que han devuelto muestras con estilo directo en la búsqueda en el corpus.
\end{abstract}

PALABRAS ClAVES: Semántica; corpus; relacionalidad; estilo directo; discursividad.

SUMARIO: 1. Introducción. 2. Los problemas del Análisis Relacional. 3. La singular gramática de los verbos discursivos. 4. Hacia una demostración empírica de la no relacionalidad y la discursividad: estudio de corpus del estilo directo en inglés. 4.1 Metodología. 4.2 Análisis de los resultados. 4.2.1 Clasificación de Hooper y Thomson (1973). 4.2.2 Clasificación de Cattell (1978). 4.2.3 Clasificación de Zwicky (1971). 5. Conclusiones.

\section{THE RELATION BETWEEN DISCURSIVITY AND NON-RE- LATIONALITY: A CORPUS- BASED ANALYSIS}

ABSTRACT: In English there is a group of complement verbs that behaves differently from the rest regarding its semantics and syntax. The aim of this paper is to determine the nature of this behaviour. Our hypothesis is that the specificity of these verbs is related to their reference to discourse, thus they are called discursives. To empirically demonstrate it, we have analysed a corpus-based study of complement verbs that can co-occur with direct speech constructions. First of all, complement verbs have been chosen from the most important taxonomies in literature to query them followed by a direct quote in the British $\mathrm{Na}$ tional Corpus. Results have confirmed our hypothesis because only discursive verbs have samples with direct speech in the corpus.

KEY WORDS: Semantics; corpus; relationality; direct speech; discursivity.

SUMMARY: 1 . Introduction. 2. Problems of the Relational Analysis. 3. The special grammar of discoursive verbs. 4 . Towards an empirical evidence of discursivity and non relationality: Corpus-based study of direct speech in English. 4.1 Methodology. 4.2 Analysis of results. 4.2.1 Hooper and Thompson's taxonomy (1973). 4.2.2 Cattell's taxonomy (1978). 4.2.3 Zwicky's taxonomy (1971). 5. Conclusions.

\section{LA RELATION ENTRE LA DISCURSIVITE ET LA NON RELATIONNALITE: UNE ANALYSE FONDEE SUR DES CORPUS}

RÉSUMÉ: En anglais il y a un groupe de verbes complétifs qui se comportent de manière différente du reste en ce qui concerne la sémantique et la syntaxe. L'objectif de cette étude est de déterminer la nature de ce comportement. Notre hypothèse est que les spécificités de ces verbes dérivent de leurs référence au discours, de ce fait, ils sont appelés discursifs. Pour démontrer empiriquement cette hypothèse nous avons fait une analyse fondée sur un corpus des verbes complétifs qui peuvent apparaître avec des construction de style direct. Avant tout, nous avons choisi les verbes appartenant aux classifications les plus importantes dans la littérature, pour ensuite les contraster avec les verbes suivis du style direct trouvés dans le British National Corpus. Les résultats confirment notre hypothèse car seuls les verbes discursifs nous offrent des exemples avec le style direct dans la recherche de corpus.

MOTS CLÉS: Sémantique; corpus relationnalité; style direct; discursivité.

SOMMAIRE: 1. Introduction. 2 Problèmes de l'analyse relationnelle. 3. La grammaire singulière. 4. Vers une démostration empirique de la relation de la discursivite et la non relationnalité: étude de corpus au style direct en anglais. 4.1 Méthodologie. 4.2 Analyse des résultats. 4.2.1 Classification de Hooper et Thompson (1973). 4.2.2 Classification de Cattell (1978). 4.2.3 Classification de Zwicky (1971). 5. Conclusions.
Fecha de Recepción 


\title{
La relación entre la discursividad y la no relacionalidad: un análisis basado en corpus
}

\author{
AROA ORREQUIA BAREA
}

\section{INTRODUCCIÓN}

Al igual que en muchas otras lenguas, encontramos entre los verbos del inglés que subordinan cláusulas completivas un grupo con un comportamiento especial en lo que a su semántica y sintaxis se refiere. De manera general, se considera que los verbos completivos son transitivos, es decir, que toman una cláusula como complemento porque expresan una relación entre un individuo -el sujeto- y la proposición, que denota el complemento oracional ${ }^{1}$. Sin embargo, algunos verbos completivos no establecen una relación entre el sujeto de la oración y el objeto subordinado por el verbo. Este último grupo de verbos se conoce en la literatura como verbos no relacionales (por oposición a los relacionales que son los que sí establecen dicha relación). Será en este último grupo de verbos en los que centraremos nuestra atención en este trabajo. Partiremos de la hipótesis de que el comportamiento especial que tienen estos verbos se debe a su referencia al discurso. Este es el motivo por el que los denominaremos, de aquí en adelante, verbos discursivos. Para demostrar empíricamente nuestra hipótesis, realizaremos búsquedas en el British National Corpus (de aquí en adelante BNC) de muestras de estos verbos con estilo directo, máxima representación del discurso.

\section{LOS PROBLEMAS DEL ANÁlisis Relacional}

Los verbos completivos son tradicionalmente analizados en términos de lo que se conoce como el análisis relacional. Moltmann (2003: 79) resume este análisis en los dos siguientes asertos: (a) la cláusula completiva denota una proposición que actúa como uno de los argumentos del verbo principal y (b) este verbo expresa una relación entre el individuo denotado por su sujeto y la proposición que expresa la completiva. La combinación de estos dos elementos lleva a una determinada concepción del contenido semántico de las cláusulas subordinadas que no se cumple en el conjunto de los verbos completivos que aquí estamos analizando. Cada uno de los asertos plantea un problema para los verbos discursivos.

Con respecto al primer aserto, si las cláusulas that denotan proposiciones, tal y como defiende el análisis relacional, todos los verbos completivos

\footnotetext{
${ }_{1}^{1}$ En la tradición anglosajona a los verbos completivos se les denomina verbos de actitud, verbos proposicionales y verbos de actitud proposicional (Russell 1918: 227). Sin embargo, no usaremos esta nomenclatura en este trabajo dado que existen numerosos verbos que no siempre refieren a actitudes o contenidos mentales, como es el caso de los llamados verbos de modo de habla, que explicaremos más adelante.
} 
deberian concurrir con complementos de categoría nominal y denotación proposicional, tales como the proposition that $S$. Sin embargo, este aserto no siempre se cumple (Prior 1971, Asher 1987, Bach 1997, Moltmann 2003), pues solo un número reducido de verbos completivos permiten la sustitución de una cláusula that por el sintagma nominal the proposition that $S$. Moltmann (2003: 82) denomina a este fenómeno el problema de la sustitución.

(1) a. John believes/proves/infers/accepts/assumes/establishes/asserts that the earth is round.

b. John believes/proves/infers/accepts/assumes/establishes/asserts the proposition that the earth is round.

(2) a. John thinks/says/hopes/argues/claims/comments that the earth is round.

b. \#John thinks/says/hopes/argues/claims/comments the proposition that the earth is round.

Sin embargo, este sintagma no es el único que puede dar expresión a un contenido proposicional. Sustantivos como possibility, idea o los sustantivos deverbales como los gerundios pueden expresar eventos y proposiciones en inglés. Estas categorias podrian sustituir sin problema alguno a las cláusulas completivas de verbos como regret, forget o deny. No obstante, esta sustitución no es posible para las completivas de verbos como say, think o claim.

En relación con el segundo aserto del análisis relacional, también encontramos problemas. La concepción relacional de las actitudes proposicionales ha sido severamente criticada en la literatura filosófica por sus deficiencias conceptuales (Schiffer 1990, Richard 1990, Matthews 2007). El principal argumento tiene que ver con la no discernibilidad de la eventualidad denotada por el verbo completivo y el complemento oracional. Moltmann (2003: 89) señala que cuando ciertos predicados forman parte de la restricción de un cuantificador objeto de un verbo proposicional, su argumento no puede identificarse con la denotación proposicional del complemento, sino que incluye la denotación del verbo completivo.

(3) a. John said something nice (namely that S) (Moltmann 2003: 89)

b. \#?The proposition/idea that $\mathrm{S}$ is nice.

c. $\{$ John's saying that $\mathrm{S} / J o h n ' s$ claim that $\mathrm{S}\}$ is nice.

(4) John said something that made Mary upset (Moltmann 2003:90)

El contenido de (3a) se corresponde con el de (3c), no con el de (3b). Suponiendo que John dijera que Mary era una mentirosa, lo que disgustó a esta no fue que ella fuera una mentirosa (o la proposición, idea, etc. de que lo fuera) sino el hecho de que John dijera tal cosa. Es fácil comprobar que el patrón de (3) no puede extenderse a todos los verbos completivos. 
(5) a. John accepted something nice (namely that S).

b. The fact/idea that $\mathrm{S}$ is nice.

c. John's accepting that $\mathrm{S}$ is nice.

(6) John accepted something that made Mary very upset.

Como puede observarse, (5a) entraña (5b), pero no (5c). Lo que disgustó a Mary no fue el hecho de que John aceptase algo sino la cosa que aceptó. Moltman resume estos casos afirmando que en ellos el adjetivo evaluativo y la cláusula relativa predican "not just of a content, but of a content 'sustained' by the particular attitudinal expressed by the predicate" (Moltmann 2003: 89). Esto es lo que Moltmann denomina el efecto objetivador de la sustitución.

Todo esto nos da muestra de la total interrelación que existe entre la sustitución (por expresiones referenciales) y la relacionalidad: si un verbo o una determinada lectura de un verbo completivo es relacional (i.e. expresa una relación sobre o hacia un objeto como dice Moltmann 2003: 87) siempre admitirá la sustitución de su complemento oracional por una expresión referencial. En los casos de unívoca denotación no relacional, la sustitución será imposible, en línea con lo dispuesto por el principio de sustitución que comentamos anteriormente ${ }^{2}$.

Por último, es necesario señalar el especial estatus de un grupo de verbos completivos que no bloquean la sustitución de manera tan radical como los predicados que hemos analizado en esta sección. Al mismo tiempo, estos verbos expresan un tipo de relación que no es, en ningún caso, asimilable a la de los verbos que hemos catalogado como relacionales. Son los verbos que en la bibliografia se han denominado verbos de modo de habla (Zwicky 1971: 223). Estos verbos pueden tomar ciertas expresiones referenciales como complemento, pero no traducen el contenido de las construcciones completivas a las que sustituyen. Dado su carácter no relacional, incluiremos estos verbos en la clase de verbos discursivos.

(7) John shouted that the files had been destroyed/a warning\}

(8) Meg whispered \{that the meal was succulent/a compliment\}

(9) He mumbled \{that he would make their life miserable/a threat\}

En resumen, los verbos completivos que denominamos discursivos: (a) no permiten que sus complementos sean expresados por expresiones referenciales y (b) no denotan una relación entre su sujeto y el contenido de su

\footnotetext{
2 Nos referimos a verbo y a lecturas porque existen algunos casos en los que los verbos completivos son ambiguos entre una lectura relacional y otra no relacional. Esto es lo que ocurre con believe. El Oxford English Dictionary recoge los dos significados siguientes:

(i) accept that (something) is true, especially without proof: The superintendent believed Lancaster's story.

(ii) hold (something) as an opinion; think: I believe we've already met.

De este modo, solo la primera acepción de believe podrá admitir expresiones referenciales:

(iii) The superintendent believed the rumour/story that Lancaster was innocent.
} 
cláusula completiva que pueda asimilarse a la que encontramos en otros verbos transitivos (incluidos los verbos completivos no discursivos).

\section{LA SINGULAR GRAMÁTICA DE LOS VERBOS DISCURSIVOS}

Como cabe esperar de cualquier fenómeno semántico de naturaleza composicional, la distinta relacionalidad de los verbos completivos tiene como correlato un comportamiento gramatical netamente diferenciado. Los verbos que en la sección anterior hemos caracterizado como discursivos y no discursivos se oponen entre sí en otros aspectos. Existen en la lengua inglesa numerosas construcciones gramaticales que se alinean con el fenómeno de la no relacionalidad, i.e. que distinguen a verbos discursivos y no discursivos, como las construcciones parentéticas, las pasivas ascendentes, la anáfora oracional, la omisión del complementante o la extracción de adjuntos (Orrequia-Barea 2017). Estas diferencias tienen que ver con el comportamiento matricial (i.e. propio de una cláusula matriz) de las completivas subordinadas a verbos discursivos. Es por esta razón que, siguiendo la terminología de Dayal y Grimshaw (2009: 14) denominaremos completivas semisubordinadas a las cláusulas completivas de verbos discursivos.

El hecho de que las completivas semisubordinadas tengan un comportamiento matricial, permite que ocurran en sus entornos fenómenos gramaticales propios de cláusulas matriciales, como por ejemplo, las transformaciones radicales (Emonds 1970: 6). Las transformaciones radicales o matriciales son operaciones sintácticas restringidas al ámbito de la cláusula principal, o lo que es lo mismo, no ocurrentes en contextos subordinados. Sin embargo, según Hooper y Thompson (1973: 472), el ámbito de las transformaciones radicales no está correctamente definido, pues también ocurren en un conjunto restringido de subordinadas completivas. Este es el motivo por el que Dayal y Grimshaw (2009: 14) proponen la terminología completivas semisubordinadas (quasi-subordinated complement clauses) para dar cuenta del carácter ambiguo de estas cláusulas, pues están subordinadas pero permiten fenómenos característicos de cláusulas matriciales.

La división que resulta de la licitación o no de las transformaciones radicales tanto estructurales como no estructurales (Heycock 2006: 181) por parte de los verbos completivos se corresponde con la división expuesta anteriormente, esto es, discursivos y no discursivos. De esta forma, los verbos discursivos permiten todo tipo de transformaciones radicales (anteposición del sintagma verbal, anteposición del constituyente negativo, dislocación a la izquierda o a la derecha, topicalización, entre otros) en sus cláusulas completivas, mientras que los verbos no discursivos no permiten ninguna de ellas.

En el apartado siguiente documentaremos una generalización empírica de gran relevancia para el estudio de la subordinación completiva que nos llevará a postular que todos los fenómenos asociados con los verbos completivos no relacionales derivan de su carácter discursivo. Concretamente, 
la generalización refiere al hecho de que solo los verbos discursivos pueden tomar complementos de estilo directo, también llamados citas directas.

\section{HACIA UNA DEMOSTRACIÓN EMPÍRICA DE LA NO RELACIONALIDAD Y LA DISCUR- SIVIDAD: ESTUDIO DE CORPUS DEL ESTILO DIRECTO EN INGLÉS}

En este apartado vamos a proponer y a analizar una construcción que parece dividir de manera transversal la clase de los verbos completivos. Para ello, hemos extraído y analizado muestras del BNC que nos permitirán una investigación empírica de textos completos emitidos en contextos auténticos, con unos sentidos y propósitos comunicativos específicos (Parodi 2008: 96). Los motivos fundamentales por los que hemos escogido este corpus han sido, por un lado, porque es uno de los más utilizados en lingüística de corpus del inglés y, por otro, porque es un corpus equilibrado y su utilidad para la descripción de la lengua inglesa ha sido probada a lo largo de los años por numerosas investigaciones. Utilizaremos el corpus como fuente de ejemplos ${ }^{3}$ para comprobar la gramaticalidad o agramaticalidad de las construcciones en estilo directo con un conjunto de verbos. De este modo, obtendremos una clasificación binaria entre los verbos que sí aparecen con dicha construcción y los verbos que no la admiten. El objetivo de este análisis es demostrar de manera empírica que existe un grupo de verbos completivos que tienen una relación con el discurso.

Hasta ahora, hemos discutido que existen dos grupos de verbos diferenciados por su capacidad de tomar o no expresiones nominales que reemplacen correctamente sus complementos oracionales sin dar lugar a agramaticalidad o a una pérdida o cambio de significado (Moltmann 2003: 86). Sin embargo, existe una construcción que, aunque no sea de naturaleza nominal, puede reemplazar de manera correcta y sin pérdida o cambio sustancial de sentido las cláusulas subordinadas. Nos referimos a la complementación en estilo directo.

\subsection{Metodología}

Para recabar el corpus que nos ha servido como fuente de ejemplos para este estudio, hemos seleccionado en la página web http://www.natcorp.ox.ac.uk/ la Universidad de Lancaster porque su interfaz nos permite descargar las muestras encontradas en la búsqueda y, además, reconoce el uso de comillas, lo cual resulta fundamental para la confección de un corpus de muestras de uso del estilo directo.

Para la operativa de búsqueda, hemos seguido la siguiente metodología. En primer lugar, de entre las taxonomias de verbos completivos existentes

\footnotetext{
${ }^{3}$ Existe actualmente un debate en torno al hecho de si esta rama de la lingüistica es una disciplina o una metodología (McEnery y Wilson 1996, Kennedy 1998, Stubbs 2001).
} 
hemos seleccionados las más relevantes en la literatura. Analizaremos los verbos asertivos y no asertivos de Hooper y Thompson (1973) y Hooper (1975), los stance y nonstance de Cattell (1978) y los que caen dentro de la definición de verbos de modo de habla de Zwicky (1971). Tomados en su conjunto, estas clasificaciones ofrecen un conjunto representativo de los verbos completivos de la lengua inglesa actual.

En segundo lugar, una vez seleccionados solo los verbos completivos, hemos procedido a la búsqueda de ejemplos en el BNC. Hemos restringido la búsqueda a verbos en forma de pasado, y ello por dos motivos: (a) la mayoría de las fuentes son textos escritos en los que se suele usar la narración, lo que comporta un uso frecuente de verbos en pasado y (b) la morfología de pasado en verbos regulares (i.e. ed), e incluso la de algunos irregulares, coincide con la del participio. Buscando verbos en esta forma, por tanto, abarcamos no solo las muestras en pasado simple sino también en otros tiempos tales como los perfectos. En algunos casos, como el del verbo tell, hemos hecho una búsqueda adicional añadiendo un dativo, pues si se busca exclusivamente a partir de la forma de pasado, solo nos aparecen muestras de pasiva. Como el resto de verbos estaban en voz activa, decidimos introducir el objeto para obtener resultados en voz activa.

En tercer lugar, para la búsqueda de muestras en estilo directo, hemos utilizado la siguiente sintaxis del motor de búsqueda: [verbo \, "] ("verbo" "espacio" "barra" "coma" "espacio" "comillas dobles"). En esta expresión, la barra sirve para para que tome la coma como un signo de puntuación y no como una expresión regular. Por su parte, la coma se emplea para separar el estilo directo del verbo subordinante.

Dado que las comillas no realizan distinción entre apertura o cierre de cita, esta página web nos proporciona, una vez realizada la búsqueda, la opción de elegir entre si las comillas son abiertas o cerradas (frequency breakdown). En esta pestaña se nos proporciona no solo la opción de elegir el tipo de comillas, sino también el número de ocurrencias de una y otra opción, y el porcentaje que ocupa cada opción con respecto al total. Por ejemplo, si buscamos: [said \, "] y clicamos en la opción de frequency breakdown, [said '] aparece con 10781 muestras que ocupan un 98'65\% del total y [said '] con 148 muestras, lo que supone el 1'35\% del total. Obviamente, en nuestro caso hemos seleccionado la primera opción, dado que buscamos verbos que tomen como su objeto complementos en estilo directo (no verbos completivos que se encuentren al final de una cita directa). En el caso del verbo tell, como decíamos, hemos necesitado introducir un objeto en el motor de búsqueda. Para ello hemos utilizado la sintaxis [told * \, "], donde el asterisco es un comodín y permite la aparición de cualquier palabra que ocurra en el entorno descrito. En la mayoría de los casos los resultados devueltos incluían el tipo de elemento que buscábamos, esto es, un objeto indirecto.

En la sección que sigue vamos a proceder al análisis de los resultados comparándolos con las clasificaciones de donde proceden los verbos en cada 
caso. El procedimiento que vamos a seguir será siempre el mismo. Partiendo de nuestra hipótesis de que todos los verbos discursivos toman complementos en estilo directo, comprobaremos si los verbos que aparecen con estilo directo en el corpus son efectivamente discursivos. Dado que estas taxonomías buscan, en distinta forma y grado, dar cuenta del especial comportamiento de los verbos completivos (asertivos, stance, etc.), analizaremos en cada caso qué clasificación resulta más efectiva, la que sustenta la taxonomía o la que divide a verbos discursivos de verbos no discursivos.

\subsection{ANÁlisis DE LOS RESULTADOS}

Los resultados del corpus analizado se basan en una búsqueda de 125 verbos completivos. Estos verbos han sido extraídos de las taxonomías y clasificaciones elaboradas en los trabajos sobre el tema que más influencia han tenido en el campo de estudio. Son muchas las teorias que han intentado dar una explicación al comportamiento especial de estos verbos proponiendo clasificaciones de verbos en base a criterios especificos. Muchos autores han indagado en las propiedades léxico-semánticas de los verbos completivos (Kiparsky y Kiparsky 1970, Hooper y Thompson 1973, Cattell 1978), algunos en las propiedades semánticas de las cláusulas completivas (Haegeman 2003, Ürögdi 2012) y otros en las de verbos y cláusulas (Meinunger 2006, Krifka 2014). En las siguientes secciones vamos a presentar los resultados que hemos obtenido en la búsqueda en el corpus en relación con cada una de las clasificaciones que hemos encontrado más relevantes en la literatura. De esta forma, podremos contrastar dichas clasificaciones con nuestros resultados para constatar de manera empírica que todos los verbos que venimos llamando discursivos aparecen con complementos en estilo directo.

\subsubsection{Clasificación de Hooper y Thomson (1973)}

Hooper y Thompson (1973: 471-481), y luego Hopper (1975: 92), proponen una clasificación de verbos en torno al concepto de 'aserción'. El criterio que propicia la división entre asertivos es el de prominencia informativa. Esto es, ciertos predicados tienen la capacidad de cambiar el foco oracional de las oraciones que lo llevan por defecto -las principales- a las subordinadas. Por tanto, los predicados asertivos permitirán la afirmación de sus complementos, mientras que los no asertivos la bloquearán. Hooper (1975: 92) además incluye en su clasificación los factivos (Kiparsky y Kiparsky 1970: 143), que implican la presuposición de sus complementos y, por tanto, bloquean la aserción. En la clasificación también aparecen los verbos semifactivos, que constituyen un grupo de verbos un tanto especial, pues la presuposición de sus complementos no es constante y comparten ciertos rasgos con los verbos asertivos, como por ejemplo, la capacidad de ocurrir en construcciones parentéticas. 
En lo que se refiere a la gramática de los verbos discursivos, Hooper y Thompson (1973: 472) y Hooper (1975: 97) relacionan el hecho de que los fenómenos matriciales ocurran en ciertas subordinadas con el componente asertivo de sus verbos completivos. Para nosotros, los fenómenos matriciales están relacionados con la discursividad, tal y como la definimos en el apartado 3 (i.e. como no relacionalidad) y como se manifiesta en los fenómenos matriciales (Orrequia-Barea 2017). En concreto, Hooper y Thompson (1973: 496) argumentan que los factivos no ocurren en estos entornos, debido a que no permiten que sus complementos sean afirmados. Por el contrario, los verbos semifactivos sí permiten que sus completivas sean afirmadas y, por tanto, para Hooper y Thompson (1973: 472), permiten la aparición de fenómenos matriciales, aunque no todos, $y$, especificamente, rechazan los de más alto nivel estructural. Nuestra expectativa en relación con estos verbos es, por tanto, que no aparezcan con complementos en estilo directo.

En relación con las búsquedas realizadas en el $\mathrm{BNC}^{4}$, la primera conclusión que cabe extraer es que existe un alto nivel de compatibilidad entre verbos asertivos, en el sentido de Hooper y Thompson, y la complementación de estilo directo. Algunos de los verbos que hemos encontrado con estilo directo son answer, explain, remark, reply, say o think ${ }^{5}$. Por supuesto, cabe extraer la misma conclusión en relación con el concepto de discursividad que aquí defendemos: todos los verbos asertivos son no relacionales y, por tanto, discursivos.

Esta conclusión puede y debe ser matizada, ya que encontramos casos aislados pero significativos de verbos asertivos que no aparecen con complementación en estilo directo en el corpus. Es el caso, por ejemplo, de los verbos hint y mention que, a pesar de tener denotación de un evento comunicativo, no encajan con nuestra definición de verbos discursivos. Aunque no presupongan la verdad de sus complementos, requisito que plantea Hooper para ser asertivos, no permiten fenómenos matriciales de ningún tipo en su entorno.

(10) * (When asked about John) Peter (only) hinted/mentioned that Bill, Mary had visited him.

(11) \#Peter hinted/mentioned that Mary had fortunately visited Bill.

Como en el resto de los casos analizados en este trabajo, tanto hint como mention permiten que sus complementos sean reemplazados por expresiones referenciales sin ningún cambio o pérdida de significado.

(12) Peter \{hinted at/mentioned\} \{Mary's visit to Bill/a visit to Bill by Mary\}

\footnotetext{
4 Excluimos de nuestro análisis los predicados compuestos de be que aparecen en la clasificación de Hooper y Thompson. Estos predicados no aparecen con estilo directo y, en consonancia con nuestras predicciones, son analizados como no asertivos por Hooper y Thompson (1973: 478).

5 Para una ampliación de nuestra clasificación, véase, al respecto, anexo.
} 
En resumen, estos verbos no son discursivos y, de hecho, el corpus nos muestra su incapacidad para concurrir con complementación en estilo directo, lo que refuerza la conclusión de que el concepto de asertividad no resulta totalmente adecuado para dar cuenta de los fenómenos gramaticales propios de los verbos discursivos. Por la misma razón, el concepto de verbo completivo discursivo sí resulta eficaz a la hora de prever la distribución de estos fenómenos.

Hay un último dato que refuerza de manera indirecta esta misma conclusión. La clase de los asertivos fuertes de Hooper y Thompson (1973: 474478) distingue entre verbos que denotan proferencias (Clase A) y actitudes mentales (Clase B). Según nuestro corpus de ejemplos, ambos grupos de verbos aparecen con estilo directo. Esto demostraria que el estilo directo, y la referencia al discurso que manifiesta, no es una propiedad exclusiva de verbos relacionados con la producción real de enunciados (Clase A), sino también de verbos que refieren a la producción potencial de enunciado (Clase B).

Con respecto a los verbos semifactivos, las búsquedas en el corpus ofrecen un panorama más complejo que el que habiamos encontrado hasta el momento. Los verbos semifactivos no toman cláusulas completivas semisubordinadas. Según la hipótesis que manejamos en este trabajo, estos verbos no deberian aparecer con complementación en estilo directo. Sin embargo, los resultados que nos ofrece el corpus se corresponden parcialmente con esta expectativa. Efectivamente, encontramos verbos como discover, find out, know, learn, notice, perceive y see que no van acompañados nunca de cláusulas en estilo directo según el corpus que hemos consultado. Al mismo tiempo, encontramos un grupo de verbos semifactivos, siguiendo a Hooper y Thompson (1973: 482), que sí aparecen con estilo directo. Es el caso de note, observe, recall, remember y reveal. Para que la hipótesis que sostenemos pueda tener vigencia, necesitamos una explicación de estos casos, explicación que puede hallarse en el carácter ambiguo de los verbos semifactivos del segundo grupo.

Todos los verbos semifactivos que aparecen con estilo directo en nuestro corpus son ambiguos entre una lectura puramente semifactiva y otra discurisva, o relacionada con un acto comunicativo. Este dato es fácil de constatar recurriendo a la información lexicográfica sobre estos predicados. Todos estos verbos tienen dos acepciones en el Oxford English Dictionary 6 (OED de aquí en adelante): (i) una semifactiva, generalmente aludiendo a procesos mentales relacionados con estados de cosas consideradas como factuales por el hablante; (ii) refieren sistemáticamente al uso de un enunciado con fines comunicativos. Se da la circunstancia de que en esta segunda acepción los verbos no son realmente factivos. Por ejemplo, el hablante que dice (13a)

\footnotetext{
${ }^{6}$ Todas las acepciones y ejemplos extraídos del OED se aplican al período actual.
} 
o (13b) no tiene por qué participar en la verdad del complemento de estilo indirecto o directo, respectivamente.

(13) a. It has been observed by ancient writers that the second bridge was a very good bridge (tomado del OED).

b. In his essay he observed, "When we set before our eyes a round globe... it is certain that the idea thereby imprinted in out mind is of a flat circle." (tomado del BNC).

Cabe concluir, a partir de esta ambigüedad léxico-semántica, que estos verbos no son semifactivos en todas sus lecturas y que, de hecho, son discursivos en al menos una de ellas. Es precisamente en estas lecturas que estos predicados admiten complementos de estilo directo. Parece claro, por tanto, que la hipótesis de que el estilo directo tiene la misma distribución que las completivas semisubordinadas no se debilita con los datos del corpus, sino que más bien queda reforzada. Solo en su lectura discursiva y no factiva es posible encontrar los verbos de la clase semifactivos de Hooper y Thompson seguidos de complementos en estilo directo. Como muestra de que las lecturas discursivas de estos verbos toman completivas semisubordinadas, vamos a considerar el contraste que se da entre (13c) y (14a).

(13) c. It has been observed by ancient writer that (fortunately) the second bridge was (fortunately) a very good bridge (adaptado del OED).

(14) a. You will also observe that (unfortunately) I (fortunately) do not have eyes in the back of my head (adaptado del OED).

El adverbio evaluativo, un fenómeno matricial no estructural, forma parte del contenido proposicional introducido por el verbo observe en (13c), como demuestra el hecho de que (13c) entraña (13d).

(13) d. It has been observed by ancient writers that it was fortunate that the second bridge was a very good bridge.

Por el contrario, (14a) no entraña (14b), lo que demuestra que el adverbio evaluativo es, en todo caso, parte del contenido presupuesto por el hablante, no por el sujeto de la cláusula principal.

(14) b. You will observe that it is unfortunate that I do not have eyes in the back of my head.

En resumen, los datos son perfectamente compatibles con la hipótesis que defendemos en este capitulo: solo los verbos discursivos, i.e. aquellos que toman complementación completiva semisubordinada, pueden subcategorizar complementos en estilo directo. 
Mención aparte merece el caso del verbo realise, de nuevo un verbo semifactivo que tiene apariciones con estilo directo en el corpus que tomamos como referencia. Obviamente, este caso es distinto al de los verbos que acabamos de comentar, puesto que realise no manifiesta ambigüedad entre una lectura semifactiva y otra discursiva. Realise, clasificado por Hooper y Thompson (1973: 480) como un verbo semifactivo, no puede aparecer con nominales complejos del tipo the fact that $S$.

(15) John realised *(the fact that) the defendant was not telling the truth.

Este hecho hace que realise quede descartado de la clase de los verbos no discursivos tal y como los hemos definido en este trabajo. Además, este hecho se correlaciona con una gramática semisubordinada, pues permite tópicos sintácticos.

(16) Bill realised that, the necklace, Fred had given to Jeannette before he met Alice. (tomados de Ernst 2002: 408).

Por tanto, y con respecto a nuestros criterios de clasificación, podemos decir que realise pertenece a los verbos que venimos denominando discursivos en este trabajo, y que, en consecuencia, admite la complementación de estilo directo. Verbos como observe se comportan del mismo modo, por lo que también pertenece a la clase de verbos discursivos.

Con respecto a los verbos no asertivos, como deny y doubt, y a los puramente factivos, como accept o regret, el corpus nos devuelve los resultados que esperábamos: son absolutamente relacionales y, por tanto, no discursivos, esto es, el estilo directo no es posible nunca en estos casos.

En conclusión, podemos decir que la clasificación de Hooper y Thompson (1973: 471-481), en términos de aserción, tiene bastantes puntos en común con la que aquí sostenemos en términos de denotación discursiva. No obstante, las dos clasificaciones difieren de manera global en relación con la clase de los semifactivos, que son asertivos para Hooper y Thompson pero no discursivos por el criterio de relacionalidad y por los fenómenos matriciales. Lo mismo ocurre con ciertos verbos aislados, como hint y mention. En todos estos casos, la complementación de estilo directo coincide extensionalmente con la denotación discursiva, pero no con la asertiva (en el sentido de Hooper y Thompson) de los verbos completivos.

\subsubsection{Clasificación de Cattell (1978)}

En la literatura, hay un conjunto de teorias que se asocian con el concepto de acervo o terreno común (CG de aquí en adelante). Cattell (1978: 77) realiza una clasificación de verbos de acuerdo con la pertenencia o no de la información de la proposición subordinada al CG. En este sentido, los verbos se dividen en stance, que pueden cambiar el CG, ya sea mediante la 
incorporación de nuevas proposiciones (verbos volunteered stance) o reforzando/debilitando las proposiciones que ya pertenecen al CG (verbos response stance). Los verbos nonstance no realizan ningún cambio en el CG. Si comparamos esta clasificación con la de Hooper (1975: 92), los verbos stance, tanto volunteered como response, se corresponderian en su gran mayoría con los asertivos. Los nonstance, en cambio, se corresponden con los no asertivos y los factivos. Veamos cuáles son los resultados obtenidos en el corpus en relación con esta clasificación.

El nivel de coincidencia entre nuestra clasificación y la de Cattell (1978: 77) es menor que el que encontramos en la clasificación de Hooper y Thompson (1973: 471-481). En primer lugar, los verbos nonstance se corresponden en gran medida con los no asertivos de Hooper y Thompson (i.e. factivos y semifactivos). En nuestra clasificación, estos verbos son no discursivos, o relacionales, pues permiten nominales complejos como objetos, cf. (17a) y (18a) y no licitan fenómenos matriciales como la topicalización temática (17b) y (18b).

(17) a. John regretted the fact that the defendant had told the truth.

b. *John regretted that the truth, the defendant had told.

(18) a. John doubted the fact that Mary had passed the exam.

b. *John doubted that the exam, Mary had passed.

En consonancia con ellos, el corpus no nos devuelve ningún registro de la mayoría de estos verbos con estilo directo. Sin embargo, encontramos dentro de este grupo algunos verbos que aparecen con citas directas en el BNC. Es el caso de comment o point out ${ }^{7}$. Estos datos encajan perfectamente con nuestra clasificación, puesto que los verbos en cuestión son discursivos. Por ejemplo, no admiten nominales complejos y permiten fenómenos matriciales de alto nivel, como la topicalización temática.

(19) a. *Susan commented the fact that Sarah didn't accept the invitation.

b. Susan commented that the invitation, Sarah didn't accept.

(20) a. *The judge pointed out the fact that she had committed a crime.

b. The judge pointed out that a crime, she had committed.

Por otra parte, nos encontramos con el verbo mention clasificado como nonstance. Dado lo comentado en la sección anterior, esta es la clasificación correcta, pues mention es relacional y, por tanto, no discursivo, y no concurre con fenómenos matriciales, como muestran (10)-(12) repetidas aquí ahora como (21) y (22).

(21) *(When asked about John) Peter (only) mentioned that Bill, Mary had visited him.

\footnotetext{
7 Para una ampliación de nuestra clasificación, véase, al respecto, anexo.
} 
(22) \#Peter mentioned that Mary had fortunately visited Bill.

(23) a. Peter mentioned that Mary had visited Bill.

b. Peter mentioned \{Mary's visited to Bill/a visit to Bill by Mary\}

De manera general, los grupos volunteered y response stance recogen los verbos asertivos de Hooper y Thompson (1973: 474-479), por lo que, se aplica lo dicho sobre esta clase en el apartado anterior. El resultado más importante es que, de nuevo, la gran mayoría de verbos de estos grupos toman citas directas. Hay, no obstante, excepciones significativas. En el grupo de los response stance nos encontramos con dos verbos no asertivos, accept y deny. Para nosotros, ninguno de estos verbos es discursivo.

(24) a. She accepted (the idea) that she needed a tracheotomy to live.

b. *She accepted that, a tracheotomy, she needed to live.

(25) a. The teacher denied (the fact) that the pupils had failed the test.

b. *The teacher denied that, the test, the pupils had failed.

Como era de esperar, el corpus no muestra registros de concurrencia con estilo directo para ninguno de estos verbos.

En conclusión, los resultados de la búsqueda de los verbos de Cattell (1978: 77) confirman, de nuevo, nuestra hipótesis de que solo los verbos discursivos toman complementos de estilo directo.

\subsubsection{Clasificación de Zwicky (1971)}

El último grupo de verbos que vamos a verificar en esta sección son los verbos de modo de habla (Zwicky 1971: 223). De acuerdo con Zwicky, el criterio que los distingue del resto es que son "verbs referring to intended acts of communication by speech and describing physical characteristics of the speech act. Hence the label manner-of-speaking verbs is appropiate" (Zwicky 1971:223).

Como ya comentamos, estos verbos no se consideran en los estudios sobre los verbos completivos como verbos de actitud o proposicionales (Russell 1918: 227). Es quizás por esta razón que solo aparecen de manera testimonial en las clasificaciones de verbos completivos que hemos revisado anteriormente. Por supuesto, cabe poca duda de que estos predicados pueden tomar complementos oracionales del mismo tipo que verbos como think o believe. Es por ello que hemos considerado importante incluirlos en nuestro trabajo de campo.

Además de los verbos que aparecen en el trabajo de Zwicky, hemos decidido ampliar dicho grupo recurriendo a otros trabajos que, aunque no se centran en la explicación de dichos verbos, proporcionan un gran número de ejemplos (Erteschik-Shir 1973, Cinque 1990, Rojo y Valenzuela 2001, Mastrofini 2014). El punto en común que tienen todos estos verbos es que denotan un acto comunicativo, pero más concretamente un acto discursivo. 
El hecho de que estos verbos denoten eventos discursivos hace que esperemos que, en base a todos los hechos vistos anteriormente, ocurran de manera sistemática con estilo directo.

De manera general, se cumple nuestra predicción: estos verbos aparecen con estilo directo ${ }^{8}$. La explicación intuitiva es que tienen una relación muy directa con el discurso. Para nosotros y como ya se dijo, esto se traduce en que estos verbos no admiten la sustitución de sus cláusulas subordinadas por nominales complejos y permiten todo tipo de fenómenos matriciales subordinados en sus completivas.

(26) a. Mary shouted (*the idea/content) that she was going to pay the dinner.

b. Mary shouted that the dinner she was going to pay for.

Este tipo de ejemplos demuestra que estos verbos pertenecen a nuestra clase de verbos discursivos. Estos verbos también pueden ocurrir con nominales seguidos de estilo directo.

(27) Mary shouted those words, "I'm going to pay the dinner".

Estas construcciones no cuentan como casos de sustitución. Estos complementos nominales no son los mismos que sustituyen a las completivas de los verbos no discursivos, pues no tienen la capacidad de parafrasear el contenido de los complementos oracionales reemplazados. De hecho, estos verbos no funcionan con cualquier nominal complejo, como hacen los verbos relacionales, sino que solo aceptan nominales que denoten un acto de habla o un enunciado (e.g. those words, threat o warning).

En este sentido, la conclusión que cabe extraer de los resultados proporcionados por el corpus es que los verbos de modo de habla son plenamente discursivos por los criterios que hemos establecido en este trabajo.

\section{Conclusiones}

En este trabajo hemos llevado a cabo un estudio en profundidad de un grupo de verbos completivos y sus complementos oracionales que no encuentran una explicación satisfactoria dentro de una visión relacional de la transitividad completiva. La concepción de los complementos como entidades abstractas y de los verbos como relaciones se encuentra con el problema empírico de la sustitución. A ello se suma todo un conjunto de fenómenos gramaticales que divide a los verbos completivos en relacionales y no relacionales.

\footnotetext{
8 Para una ampliación de nuestra clasificación, véase, al respecto, anexo.
} 
En primer lugar, hemos compilado y sistematizado unas estructuras gramaticales (Orrequia-Barea 2017) que nos han permitido establecer y refinar dos clases de verbos bien diferenciadas: los discursivos y los no discursivos. En segundo lugar, el uso del corpus como fuente de ejemplos ha confirmado la hipótesis de que la semisubordinación completiva tiene el mismo patrón distribucional que la complementación en estilo directo. Motivados por la justificada crítica de que la visión citativa es dificilmente aplicable a todos los casos de subordinación completiva, hemos ofrecido un conjunto de resultados y una clasificación que apuntan a un claro paralelismo entre la denotación discursiva de los verbos completivos (o, lo que es lo mismo, el carácter semisubordinante de sus complementos oracionales) y la posibilidad de concurrir con complementación en estilo directo.

La validez teórica de los resultados que ofrece es clara por cuanto constituye una auténtica generalización descriptiva sobre la lengua inglesa: solo los verbos discursivos, definidos como aquí lo hemos hecho (no relacionalidad, fenómenos matriciales, etc.) concurren con citas directas en el inglés actual. Esta, podemos ahora afirmar, es una importante generalización descriptiva complementaria de otra más conocida: los verbos no discursivos son los que permiten la expresión de sus complementos proposicionales a través de una categoria nominal. Estas dos generalizaciones están conectadas y, dados los objetivos de nuestro trabajo, confirman nuestra hipótesis de que el elemento que determina el patrón gramatical de la semisubordinación es la referencia al discurso.

\section{Anexos}

\begin{tabular}{|l|l|}
\hline $\begin{array}{l}\text { Verbos que permiten complementa- } \\
\text { ción en estilo directo }\end{array}$ & $\begin{array}{l}\text { Verbos que no permiten comple- } \\
\text { mentación en estilo directo }\end{array}$ \\
\hline Acknowledge & Accept \\
\hline Add & Affirm \\
\hline Admit & Amuse \\
\hline Admonish & Assert \\
\hline Agree & Assure \\
\hline Allege & Asume \\
\hline Answer & Bother \\
\hline Argue & Buzz \\
\hline Bark & Calculate \\
\hline Believe & Care \\
\hline Boast & Charge \\
\hline Claim & Contend \\
\hline Comment & Convey \\
\hline Conclude & Convince \\
\hline Confirm & Croon \\
\hline Conjecture & Deduce \\
\hline & \\
\hline
\end{tabular}




\begin{tabular}{|l|l|}
\hline Consider & Deem \\
\hline Cry & Deny \\
\hline Decide & Detail \\
\hline Declare & Discourse \\
\hline Divulge & Discover \\
\hline Exclaim & Doubt \\
\hline Explain & Email \\
\hline Feel & Emphasize \\
\hline Figure & Envisage \\
\hline Groan & Estimate \\
\hline Growl & Except \\
\hline Grumble & Expect \\
\hline Insist & Fancy \\
\hline Lament & Find out \\
\hline Mantain & Forget \\
\hline Moan & Grant \\
\hline Mumble & Guess \\
\hline Murmur & Hint \\
\hline Mutter & Hope \\
\hline Note & Imagine \\
\hline Observe & Imply \\
\hline Point out & Indicate \\
\hline Predict & Intimate \\
\hline Realise & Judge \\
\hline Recall & Know \\
\hline Remark & Learn \\
\hline Remember & Make sense \\
\hline Repeat & Mention \\
\hline Reply & Notice \\
\hline Report & Perceive \\
\hline Retort & Postulate \\
\hline Reveal & Presume \\
\hline Roar & Prophesy \\
\hline Say & Propose \\
\hline Scream & Reckon \\
\hline Shout & Recognise \\
\hline Squeak & Regret \\
\hline Stammer & Remind \\
\hline State & Resent \\
\hline Suggest & See \\
\hline Tell & Suffice \\
\hline Think & Suppose \\
\hline Wail & Surmise \\
\hline Whisper & Suspect \\
\hline & \\
\hline
\end{tabular}




\begin{tabular}{|l|l|}
\hline Write & Swear \\
\hline yell & Verify \\
\hline & Wheeze \\
\hline
\end{tabular}

\section{REFERENCIAS}

ASHER, N. (1993): Reference to $A b$ stract Objects in Discourse, Dordrecht: Kluwer Academic Publishers.

BACH, K. (1997): "Do belief reports report beliefs?", Pacific Philosophical Quarterly, 78, pp. 215-241.

The British National Corpus, version 3 (BNC XML Edition). 2007. Distributed by Bodleian Libraries, University of Oxford, on behalf of the BNC Consortium. URL: http://www.natcorp.ox.ac.uk/ (última fecha de acceso 04/12/17).

CATTELL, R. (1978): "On the source of interrogative adverbs", Language, 54, pp 61-77.

CINQUE, G. (1990): Types of A'-Dependencies, Cambridge, MA: MIT Press.

DAYAL, V. \& GRIMSHAW, J. (2009): Subordination at the interface. The quasi-subordination hypothesis, Rutgers University: manuscrito.

EMONDS, J. (1970): Root and Structure-preserving Transformations. Tesis doctoral. Cambridge, MA: MIT.

ERNST, T. (2002): The Syntax of Adjuncts, Cambridge: Cambridge University Press.

ERTESCHIK-SHIR, N. (1973): On the Nature of Islands Constraints. Tesis doctoral. Cambridge, MA: MIT.

HAEGEMAN, L. (2003): “Conditional clauses. External and internal syntax, Mind \& Language, 18, 4, pp. 317-339.

HEYCOCK, C. (2006): "Embedded root phenomena", Everaert, M. y van Riemsdijk, H. (eds.), The
Blackwell Companion to Syntax, Vol. II, Oxford: Blackwell, pp. 174-209.

HOOPER, J. (1975): "On assertive predicates", Kimball, J. (ed.), Syntax and Semantics 4, Nueva York: Academic Press, pp. 91124.

HOOPER, J. \& THOMPSON, S. (1973): "On the applicability of root transformations", Linguistic Inquiry, 4, pp. 465-497.

KENNEDY, G. (1998): An Introduction to Corpus Linguistics, Londres: Longman.

KIPARSKY, P. \& KIPARSKY, C. (1970): "Fact", Bierwisch, M. y Heidolph, K. (eds.), Progress in Linguistics, La Haya: Mouton, pp. 143-173.

KRIFKA, M. (2014): "Embedding illocutionary acts", Roeper, T. y Speas, M. (eds.), Recursion: Complexity in Cognition, Dodrecht: Springer, pp. 59-88.

MASTROFINI, R. (2014): "English manner of speaking verbs and their Italian translations: A cross-linguistic comparison", RILA: Rassegna Italiana di Linguistica Applicata 2, 2, 3, pp. 131-154.

MATTHEWS, R. (2007): The Measure of Mind: Proposition Attitudes and their Attribution. Oxford: Oxford University Press.

MCENERY, A. \& WILSON, A. (1996): Corpus Linguistics, Edimburgo: Edinburg University Press.

MEINUNGER, A. (2006): "On the discourse impact of subordinate clauses", Molnár, V. y Winkler, S. (eds.), The Architecture of Focus. Studies in Generative 
Grammar, 82, Berlín: Mouton de Gruyter, pp. 459-487.

MOLTMANN, F. (2003): "Propositional attitudes without propositions", Synthese, 135, 1, pp. 77-118.

OED Online. June 2017. Oxford University Press. http://www.oed.com/viewdictionaryentry/Entry/11125 (último acceso 10/12/2017).

ORREQUIA-BAREA, A. (2017): Semántica de los verbos proposicionales del inglés actual. Tesis doctoral inédita. Cádiz: Universidad de Cádiz.

PARODI, G. (2008): "Lingüística de corpus una introducción al ámbito", Revista de Lingüística Teórica y Aplicada, 46, 1, pp. 93-119.

PRIOR, A. (1971): Objects of thought, Oxford: Clarendon Press.

RICHARD, M. (1990): Propositional Attitudes. An Essay on Thoughts and How We Ascribe them, Nueva York: Cambridge University Press.
ROJO, A. \& VALENZUELA, J. (2001): "How to say things with words: ways of saying in English and Spanish", Meta: Journal of traducteurs, 46, 3, pp. 467-477.

RUSSELL, B. (1918): "The Philosophy of Logical Atomism", Logic and Knowledge, Londres: Routledge, pp. 187-270.

SCHIFFER, S. (1990): "The mode-ofpresentantion problem", Anderson, A. y Owens, J. (eds.), Propositional Attitudes: The Role of content in Logic, Language, and Mind, Standford: CSLI Press. Pp. 249-268.

STUBBS, M. (2001): Words and Phrases. Corpus Studies of Lexical Semantics, Oxford: Blackwell.

ÜRÖGDI, B. (2012): Operator Movement in Embedded Clauses. Tesis doctoral. Budapest: ELTE.

ZWICKY, A. (1971): "In a manner of speaking”, Linguistic Inquiry, 2, pp. 223-233. 